\title{
Intraoperative radiotherapy (IORT) induced key molecular pathways in tumor bed of breast cancer patients: a pilot study
}

\author{
M. Shahani' ${ }^{1}$ J. Shakeri', M.E. Akbari ${ }^{*}$, A. Tafti², H. Zali3 ${ }^{3}$ N. Nafisi4, \\ M. Rezaei-Tavirani ${ }^{5}$, S.H. Jamaldini Ezabady6, A. Akbari' ${ }^{1}$, M. Samsami ${ }^{1}$ \\ ${ }^{1}$ Cancer Research Center, Shahid Beheshti University of Medical Sciences, Tehran, Iran \\ ${ }^{2}$ Department of Medical Biotechnology, Faculty of Medicine, Arak University of Medical Science, Arak, Iran \\ ${ }^{3}$ School of Advanced Technology in Medicine, Shahid Beheshti University of Medical Sciences, Tehran, Iran \\ ${ }^{4}$ Breast Department, Iran University of Medical Sciences, Tehran, Iran \\ ${ }^{5}$ Proteomics Research Center, Faculty of Paramedical Sciences, Shahid Beheshti University of Medical Sciences, \\ Tehran, Iran \\ ${ }^{6}$ Department of Genetics, Islamic Azad University, Tehran Medical Sciences Branch, Tehran, Iran
}

\begin{abstract}
- Original article

\author{
*Corresponding authors: \\ M. Esmaeil Akbari, M.D., \\ E-mail: \\ profmeakbari@gmail.com \\ Revised: December 2019 \\ Accepted: January 2020 \\ Int. J. Radiat. Res., October 2020; \\ 18(4): $777-784$ \\ DOI: 10.18869 /acadpub.ijrr.18.4.777
}

Background: Radiotherapy (RT) is recommended to all patients undergoing Breast Conserving Surgery (BCS). Two strategies can be applied to irradiation, External Beam RT (EBRT) in addition, Intraoperative Radiation Therapy (IORT). The aim of this study was to introduce a protein biomarker panel related to molecular function of IORT. Materials and Methods: Six Breast Cancer (BC) patients as a pilot study were treated by 12 Gy (Boost dose) and $21 \mathrm{~Gy}$ (Radical dose). Samples tissue included Margin before IORT (MB), and Margin 24 hours After IORT (MA24 h). High-throughput technology such as Isobaric Tag for Relative and Absolute Quantitation (iTRAQ) was performed to study proteomic of IORT-treated tumor bed. Results: We classified 110 differentially expressed proteins (DEPs) as a protein biomarker panel by mapping the annotated coding region sequences to the reference canonical pathways in the KEGG database. Conclusion: The analyses of protein function and signaling pathways of these DEPs reveal a molecular response to the effectiveness of IORT.
\end{abstract}

Keywords: Intraoperative radiation therapy (IORT), proteomic.

\section{INTRODUCTION}

$\mathrm{BC}$ as the fifth leading cause of cancer death is recognized as the most common cancer among women (1). Considering the advances in early diagnosis and treatment of $\mathrm{BC}$, mortality has reduced by 38\% from 1989 to 2014 (2).The prevalence of BC was 9795 cases in Iran, and the age-adjusted standardized ratio (ASR) was 28.1 in 100000 people (3). Akbari et al. reported that the five- and ten-year survival rate for $\mathrm{BC}$ was estimated to be $81 \%$ and $77 \%$, respectively (4).

RT is recommended to all patients undergoing BCS and some of them after mastectomy. The main objective is to obtain more effective doses of radiation in the appropriate time and site without increasing the prevalence of complications. RT can provide curative treatment in nearly $40 \%$ of patients (5).In a previous study, long-term outcomes in patients with BCS and RT who were timely diagnosed, were similar to or even better than patients with mastectomy (6).

Two strategies can be applied to deliver irradiation to the cancer site. The first strategy is the use EBRT which is delivered from outside the body to the tumor site via protons, photons, or charged particles (7). The second strategy is 
IORT which delivers electron beams (IOeRT) and low kV-X-ray (IOXRT) (8).

By applying IORT during BCS, a high single dose is delivered to the site at the highest risk to fight subclinical tumor cell contamination with high precision owing to direct visualization (9). Tumor bed has been reported as the highest risk of breast tumor recurrence $(10,11)$. As a result, local recurrence can be significantly reduced by using an extra dose to the tumor bed (12).

EBRT can be replaced by accelerating partial breast irradiation, including an IORT session, due to approved several advantages including; lack of gap between surgery and RT, avoidance of long treatment duration, reduced radiation-induced toxicity, tumor bed delineation under direct palpation and visual assessments and sparing non-targeted tissues surrounding the tumor bed $(12,13)$. In addition to the delayed time of RT after tumor excision, EBRT has harmful effects, including cardiac attack, appearance of second tumors, or stimulation of tumor cell growth by neo angiogenesis and hypoxia condition (14). Notably, IORT can also help to save money, time, and $\mathrm{CO}_{2}$ emissions in some patients, inhibiting transportation (15).

According to specific eligibility criteria, IORT may be delivered either as an anticipated Boost, followed by conventional external RT to guarantee optimal dose delivery, or as an exclusive single radiation dose of Radical, corresponding to the administration of the entire sequence of conventional adjuvant RT (16, 17).

Some studies have demonstrated that the biological effects of RT vary depending on the dose and irradiation time. In this regard, study on MCF7 cells treated with 9 and 23 Gy was suggested dose-dependent gene-expression profiles that might regulate cell-fate decision in two different ways. The high-dose treatments inhibited the growth and proliferation of MCF7 cells and the post-irradiation cell traits showed a typical senescent phenotype, confirmed by senescence-SA- $\beta$-Gal activity, which increased in a dose and time-dependent manner (18). Another study on IORT-treated tumor bed suggested that functional annotation and gene ontology (GO) indicated that significant enrichment in molecular pathways on BC treatment is somehow single high dose-independent (19).

The aim of this study was to introduce a protein biomarker panel (as a pilot study) related to molecular functions of IORT-treated tumor bed. The overall goal of this project is to identify molecules related to therapeutic techniques such as IORT that can discover some of the complexities of these processes for future aspects of personalized RT.

\section{MATERIALS AND METHODS}

Six random selected BC patients (as a pilot study) entered into our study (table 1). These eligible patients had neither comorbidity / history of drug nor significant mental health disorder. Informed Consent from patients and Institutional Ethical Approval from Shahid Beheshti University of Medical Sciences was obtained with approval ID: IR.SBMU.RETECH.REC.1397.561 and approval date: 2018-05-13.

IORT delivered electron beams (LIAC linear accelerators, Sordina IOeRT Technologies S.p.A, Italy), and an Intraoperative accelerator was employed for cell irradiation (20). Patients were treated with irradiation following two separate strategies, including Boost and Radical dose treatment. The machine dose rate was adjusted to $1 \mathrm{cGy} / \mathrm{MU}$ during the irradiation.

Approximately $100 \mathrm{mg}$ of margin tissue was extracted before IORT (MB) as a normal group. Likewise, at the time of operation while the wound is open, in direct vision, irradiated tumor margin (under Boost and Radical) marked. Small piece of margin tissue with narrow pedicle sutured to thread and the end of thread exited from the wound, the skin incision closed except 5-10 mm left to open for final (24 hours after operation) sample extraction. The sample removed without any pain or disturbance and dressing applied. This sample named MA24 $\mathrm{h}$ under Boost and Radical doses.

\section{Protein extraction}

First total proteins were extracted from Int. J. Radiat. Res., Vol. 18 No. 4, October 2020 
samples with the TRIzol reagent used as supplied by Invitrogen Life Technologies. Then proteins of more than $200 \mu \mathrm{g}$ qualified also were labeled to be used for iTRAQ.

Table 1. Clinical and pathological data of six BC patients under IORT-treated tumor bed.

\begin{tabular}{|c|c|c|c|c|c|c|c|c|c|c|c|c|c|c|c|}
\hline & & & & & & Patholo & gic data & & & & & & IORT da & & \\
\hline $\begin{array}{c}\text { Pt } \\
\text { Number }\end{array}$ & Age & $\begin{array}{c}\text { Tumor } \\
\text { history } \\
\text { type }\end{array}$ & $\begin{array}{c}\text { Tumor } \\
\text { size } \\
(\mathrm{cm})\end{array}$ & $\begin{array}{l}\text { Node } \\
\text { status }\end{array}$ & $\begin{array}{l}\text { Tumor } \\
\text { grade }\end{array}$ & $\begin{array}{l}\text { ER/PR } \\
\text { status }\end{array}$ & $\begin{array}{c}\text { HER-2 } \\
\text { Expression }\end{array}$ & Ki67 & P53 & $\begin{array}{c}\text { Tumor } \\
\text { Necrosis }\end{array}$ & LVI & $\begin{array}{l}\text { Delivered } \\
\text { dose }(G y)\end{array}$ & $\begin{array}{c}\text { Irradiation } \\
\text { time } \\
\text { (second) }\end{array}$ & $\begin{array}{l}\frac{\text { Applicator }}{\text { Disk }} \\
\text { size }(\mathrm{cm})\end{array}$ & $\begin{array}{c}\text { Flap } \\
\text { depth } \\
(\mathrm{cm})\end{array}$ \\
\hline 1 & 53 & IDC & 4 & NO & 3 & $-/-$ & Neg & $\% 50$ & - & Present & $\begin{array}{c}\text { Not } \\
\text { seen }\end{array}$ & 12 & 63 & $7 / 9$ & 1 \\
\hline 2 & 42 & IDC & 1 & NO & 1 & + & Neg & $30 \%$ & + & Negative & $\begin{array}{l}\text { Not } \\
\text { seen }\end{array}$ & 12 & 55 & $5 / 6$ & 1.6 \\
\hline 3 & 39 & IDC & 2.5 & NO & 2 & $+/+$ & Neg & $\% 25$ & + & Not seen & $\begin{array}{c}\text { Not } \\
\text { seen }\end{array}$ & 12 & 58 & $6 / 9$ & 1.8 \\
\hline 4 & 63 & ILC & 1.3 & NO & 2 & +1 & Neg & $\% 15$ & - & Not seen & $\begin{array}{c}\text { Not } \\
\text { seen }\end{array}$ & 21 & 65 & $5 / 6$ & 2.3 \\
\hline 5 & 48 & IDC & 2 & NO & 2 & $+/+$ & Neg & $\% 30$ & + & Not seen & $\begin{array}{c}\text { Not } \\
\text { seen }\end{array}$ & 21 & 78 & $7 / 9$ & 2.2 \\
\hline 6 & 55 & IDC & 3 & NO & 2 & $+/+$ & Neg & $\% 30$ & + & Not seen & $\begin{array}{c}\text { Not } \\
\text { seen }\end{array}$ & 21 & 85 & $8 / 9$ & 2.5 \\
\hline
\end{tabular}

\section{Proteomics assays \\ Peptide labeling}

Peptide labeling was performed by iTRAQ Reagent 8-plex Kit according to the manufacturer's protocol. The labeled peptides with different reagents were combined and desalted.

\section{Peptide 1st dimensional fractionation}

The peptides were reconstituted with buffer A (5\% ACN, 95\% $\mathrm{H}_{2} \mathrm{O}$, adjusted $\mathrm{pH}$ to 9.8 with ammonia) and separated by a Shimadzu LC-20AB HPLC system coupled with a high $\mathrm{pH}$ RP column (5- $\mu$ m particles, Phenomenex). The peptides were separated at a flow rate of $1 \mathrm{ml} /$ min with a 60 min gradient: $5 \%$ buffer B $(5 \%$ $\mathrm{H}_{2} \mathrm{O}, 95 \%$ ACN, adjusted $\mathrm{pH}$ to 9.8 with ammonia) for $10 \mathrm{~min}, 5-35 \%$ buffer B for $40 \mathrm{~min}$, $35-95 \%$ buffer B for $1 \mathrm{~min}$, and $95 \%$ buffer B for $3 \mathrm{~min}$. The gradient was then decreased to $5 \% \mathrm{~B}$ within $1 \mathrm{~min}$ before re-equilibrating with 5\% buffer B for $5 \mathrm{~min}$. Elution was monitored by measuring absorbance at $214 \mathrm{~nm}$ and the eluted peptides were pooled as 20 fractions in a concatenation mode and vacuum dried.

\section{Peptide $2^{\text {nd }}$ dimensional fractionation}

Each fraction was re-suspended in buffer A (2\% ACN and $0.1 \%$ FA in water) and loaded onto Int. J. Radiat. Res., Vol. 18 No. 4, October 2020 a C18 trap column using an LC-20AD nano-HPLC instrument (Shimadzu, Kyoto, Japan) by the Autosampler. Then, the peptides were eluted from the trap column and separated by an analytical C18 column (inner diameter $75 \mu \mathrm{m}$ ' $20 \mathrm{~cm}, 3 \mu \mathrm{m})$ packed in-house. The gradient was run at $300 \mathrm{~nL} / \mathrm{min}$ starting from 8 to $35 \%$ of buffer $\mathrm{B}\left(2 \% \mathrm{H}_{2} \mathrm{O}\right.$ and $0.1 \% \mathrm{FA}$ in $\left.\mathrm{ACN}\right)$ for 35 minutes, increasing to $60 \%$ for 5 minutes, then maintaining at $80 \%$ B for 5 minutes, and finally returning to $5 \%$ in $0.1 \mathrm{~min}$ and keeping for 10 $\min$.

\section{Mass spectrometer detection}

Data acquisition was performed with a TripleTOF 5600 System (SCIEX, Framingham, MA, USA) equipped with a Nanospray III source (SCIEX, Framingham, MA, USA), a pulled quartz tip as the emitter (New Objectives, Woburn, MA) and controlled with software Analyst 1.6 (AB SCIEX, Concord, ON). Data was acquired with the following MS conditions: ion spray voltage of $2,300 \mathrm{~V}$, curtain gas of 30 , nebulizer gas of 15 , and interface heater temperature of $150{ }^{\circ} \mathrm{C}$. High sensitivity mode was used for the whole data acquisition. The mass ranges for MS1 were from 350 to $1500 \mathrm{Da}$. Based on the intensity in MS1 survey, as many as 30 product ion scans were collected if exceeding a threshold of 120 counts 
per second (counts/s) and with charge-state $2+$ to $5+$, dynamic exclusion was set for $1 / 2$ of the peak width (12 s). For iTRAQ data acquisition, the collision energy was adjusted to all precursor ions for collision-induced dissociation and the Q2 transmission window for 100 Da was $100 \%$.

\section{Protein identification and quantification}

The raw MS/MS data were converted into MGF format by ProteoWizard tool msConvert, and the exported MGF files were searched using Mascot version 2.3.02 (Matrix Science, London, UK). In this project against the human Uniprot database .To reduce the probability of false peptide identification, only those peptides with significant scores $(\geq 20)$ with $99 \%$ confidence were counted as identified. The IQuant software was used to quantitatively analyze the labeled peptides with isobaric tags (21). It integrates Mascot Percolator, a well performing machine learning method for re-scoring database search results, to provide reliable significance measures. In order to assess the confidence of peptides, the PSMs were pre-filtered at a PSM-level FDR of $1 \%$. Then, based on the "simple principle" (The parsimony principle), identified peptide sequences were assembled into a set of confident proteins. In order to control the rate of false-positive at the protein level, a protein FDR at $1 \%$, which is based on Picked protein FDR strategy will also be estimated after protein inference (Protein-level FDR $<=0.01$ ) (22). The protein quantification process includes the following steps: Protein identification, Tag impurity correction, Data normalization, Missing value imputation, Protein ratio calculation, Statistical analysis, and Results presentation. Proteins with 1.2-fold change and Q-value less than 0.05 were determined as differentially expressed protein. For quantification repeat analysis, we used CV to evaluate the reproducibility. CV is defined as the ratio of the standard deviation (SD) to the mean.

\section{Gene functional enrichment analysis}

Gene functional enrichment analysis was applied on DEPs. In this study, we used
KEGG pathway database by using DAVID (http:// david.abcc.ncifcrf.gov/) for functional enrichment analysis.

\section{Network analysis of DEPs}

Cytoscape network analysis represented the most important proteins based on degree (19).

\section{RESULTS}

\section{Identification of differentially expressed proteins classification}

We performed iTRAQ quantification after labeling of samples in three replicates (Boost / Radical, and MB/ MA24 h). In total, 1045410 spectrums were generated; also, 31572 peptides and 5860 proteins were identified with 1\% FDR. DEPs for each comparisons of group/treatments analyzed. We found that there were 110 DEPs (37 up regulated, 73 down regulated) as single high dose-independent, which were common in both Boost and Radical doses (table 2).

Table 2. Represented common DEPs in both Boost and Radical doses.

\begin{tabular}{|c|c|c|}
\hline Names & total & elements \\
\hline $\begin{array}{c}\text { Common } \\
\text { DEPs } \\
\text { under } \\
\text { Boost } \\
\text { and } \\
\text { Radical } \\
\text { doses }\end{array}$ & 110 & $\begin{array}{c}\text { A2M SERPINB1 NCF4 CNN1 GCA FMNL1 } \\
\text { PTPRC LMNB1 VASP SERPINA3 ALOX5 } \\
\text { BASP1 SLC2A3 LBP PTX3 LTF ATP5A1 } \\
\text { TLN1 FCGR3A LCP1 C9 MDH1 ANXA5 } \\
\text { IDH3A PEA15 CP SUCLA2 S100A9 HSPB1 } \\
\text { HRG RPL7A ANXA3 ELANE FGG ASS1 } \\
\text { LDHB FGB1 BST1 HK3 PRIM2 IDH1 ALD- } \\
\text { H6A1 DLST C5 CNDP2 ACADVL ITIH3 } \\
\text { CALB2 ITGB2 PROS1 TUFM ARHGDIB } \\
\text { YWHAG MMP8 PYGB PADI4 APOH } \\
\text { DEFA1 CYBB SPTA1 PLG LYZ 2-Sep } \\
\text { RPL23A APOA4 C4BPA SUCLG2 HIST1H1C } \\
\text { BPI PEBP1 ITIH4 ACOT1 C8A MSN } \\
\text { LGALS1 MYO6 WDR1 YWHAE BCAP31 } \\
\text { CFH CNN3 HRG MNDA MPO MYH11 } \\
\text { VWF VTN CAMP ALDH7A1 OCIAD1 RP2 } \\
\text { AZU1 HEBP2 HMGB2 ITGAM HIST1H1B } \\
\text { HIBCH RNASE3 RETN HADH PRTN3 ITIH2 } \\
\text { ANK1 CFB LCN2 SERPINC1 ctSg FLNA } \\
\text { ALDH9A1 C6 MMP9 }\end{array}$ \\
\hline
\end{tabular}

Int. J. Radiat. Res., Vol. 18 No. 4, October 2020 


\section{Network Analysis of DEPs as single high dose-independent}

We have analyzed 110 DEPs as single high dose-independent. Cytoscape network analysis represented the most important proteins based on 110 nodes, 350 edges and 6.36 as average node degree and 0.505 as avg. local clustering coefficient (figure 1).

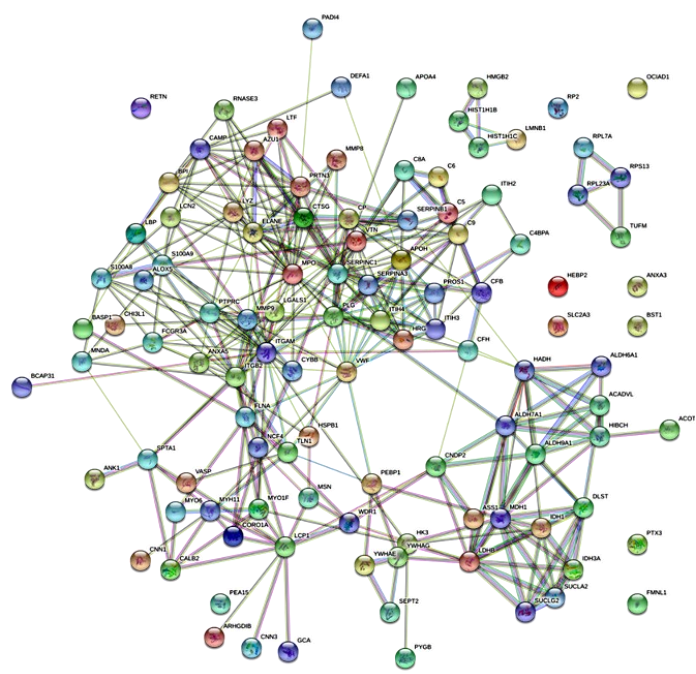

Figure1. Network analysis of 110 DEPs as single high dose-independent based on degree by string database.

According to figure 2, we identified a network of 110 DEPs as single high dose-independent. Among all, we found key proteins as a protein biomarker panel under Boost and Radical doses.

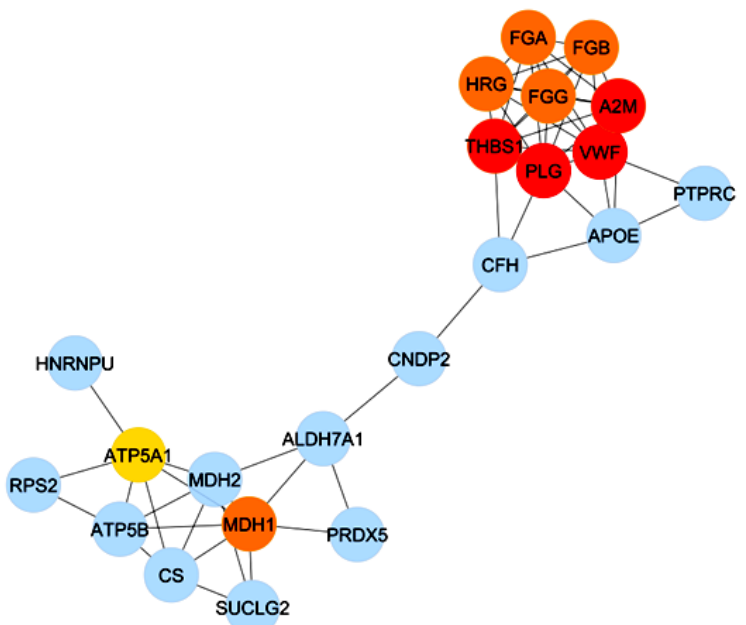

Figure 2. The deregulated densest protein-protein network (blue nodes: proteins, red nodes: proteins involved in crucial pathways) in 110 DEPs as single high dose-independent (Boost and Radical).

Int. J. Radiat. Res., Vol. 18 No. 4, October 2020
Gene ontology enrichment and functional classification 24 h post irradiation

For the better understanding of the molecular factions of IORT, we classified 110 DEPs by mapping the annotated coding region sequences to the reference canonical pathways in the KEGG pathway database (figure 3).

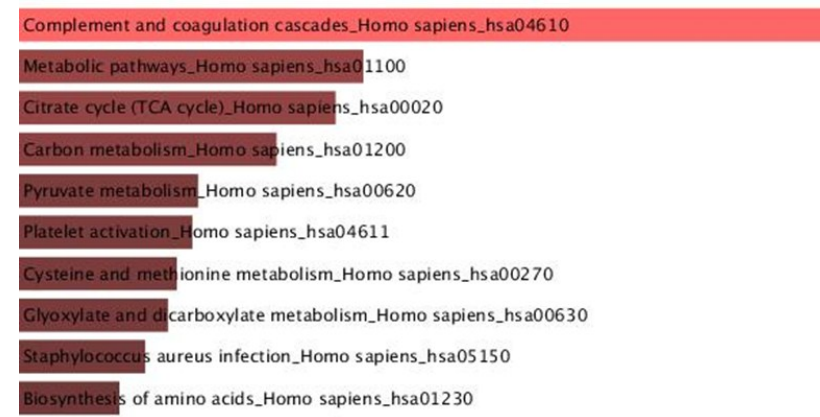

Figure 3. Biological pathways on 110 DEPs under $24 \mathrm{~h}$ after Boost and Radical doses by KEGG database.

\section{DISCUSSION}

RT is recommended to all patients undergoing BCS and some of them after mastectomy (23). Despite the technological advances made in recent decades, RT plans still advise the same total dose per organ tumor, without taking into account the biological differences attributable to the different tumor subtypes (24) . By applying IORT during BCS, a high single dose is delivered to the site at the highest risk to fight subclinical tumor cell contamination with high precision owing to direct visualization ${ }^{(9)}$. Tumor bed has been reported as the highest risk of breast tumor recurrence $(10,11)$. As a result, local recurrence can be significantly reduced by using an extra dose to the tumor bed (12).

Although researchers have shown great interest in a single high-dose clinical approaches for different cancers, limited research has examined the biological and molecular basis of single high-dose effects, especially after IORT (25). In the context of this radiation treatment modality, this study aimed to describe the molecular response, in terms of DEPs and pathways, according to the different doses of 
IORT. However, this type of investigation would need to be extended to numerous panels of samples; thus, the present work should be considered as a pilot study. Here, we report substantial alterations in proteins expression levels $24 \mathrm{~h}$ after RT in both dose (Boost and Radical). In this study proteomics approach, using ITRAQ is a necessary first step for biological pathways to describe the common molecular features associated with types of IORT. As previously described, samples were divided into two groups according to their irradiation. Then DEPs and key pathways related to RT were compared.

In this comparison, we detected 110 overlap DEPs between Boost and Radical (table 2). The identified overlapping DEPs extracted from $24 \mathrm{~h}$ post-treatment are enriched based on KEGG pathways database by using DAVID. We have analyzed 110 DEPs as single high dose-independent. Cytoscape network analysis represented the most important proteins (figure 1). All DEPs were classified into 10 protein categories (figure 2), in which the most proteins related to RT are PLG, VWF and A2M (figure 2).

As previously described by several authors, radiation effects on cells are heterogeneous and appear to act in a cell line-dependent way. This behavior was also confirmed by the pathway analysis conducted $(26,27)$. A few studies have described that, in the MCF7 cell line, the 9 Gy IR-related cell cycle, DNA replication, DNA damage response, meiotic processes, cell cycle regulation, repairing DNA strand breaks and cell survival/death balance through the activation of apoptosis signaling were deregulated after irradiation.

The Plasminogen system produced by PLG (plasminogen) plays a crucial role in physiological in addition, pathological events related to tissue regeneration, wound healing, immune response, angiogenesis, invasion and metastasis. The PLG is significantly downregulated after IORT-treated tumor bed and highly enriched in Complement and coagulation cascades.

In addition, Von Willebrand Factor Protein
(VWF) is a major platelet ligand that has been widely used, as a biomarker in cancer growth and metastasis and associated inflammation, is significantly downregulated at $24 \mathrm{~h}$ post irradiation also highly enriched in complement and coagulation cascades. As shown in figure 4, refer to complement and coagulation cascades kegg pathway data base, enrichment associated with changes in these related key pathway to RT have been illustrated which indicates that two doses of IORT (figure 3 ) is able to reduce cell growth and cell proliferation.

Functional annotation revealed new insights of IORT in BC patients. Local pathways and systemic effects; such as Complement and coagulation cascades, phagosome, metabolomics pathways and immune responses were enriched through IORT (figure 3).

Eventually, results revealed new insights of IORT in BC management; all of these biological responses in tumor cells may be towards effectiveness of IORT and it shows that although IORT is a local treatment, it can have systemic effects.

\section{CONCLUSION}

IORT can support targeted and accurate treatment of patients by modulating the surrounding tissue around the tumor. We suggest a protein biomarker panel for efficacy of IORT. However, this type of investigation would need to be extended to numerous panels of samples; thus, the present work should be considered as a pilot study

By using comprehensive techniques such as proteomic, we found the molecular basis of IORT -related changes that have already showed clinical and epidemiological effects in treating BC patients. In addition, key molecular pathways that induced by IORT indicated that, cellular microenvironment as a main cause of changes in cells through tumor development, can be affected by IORT. Due to the effectiveness of IORT, it may be recommended as a standard method for $\mathrm{BC}$ patients. 


\section{ACKNOWLEDGMENTS}

The authors thank Dr. Kui Wu; Cancer Institute, BGI-Research, China National GeneBank (CNGB) for Collaborating and for valuable comments provided on this manuscript.

\section{Conflicts of interest: Declared none.}

\section{REFERENCES}

1. Movahedi $M$, Haghighat $S$, Khayamzadeh $M$, Moradi $A$, Ghanbari-Motlagh A, Mirzaei $\mathrm{H}$, et al. (2012) Survival rate of breast cancer based on geographical variation in Iran, a national study. Iranian Red Crescent Medical Journal, 14 (12): 798.

2. Siegel R, Miller K, Jemal A (2017) Cancer Statistics CA. Cancer J Clin, 67(1): 7-30.

3. Mohebbi E, Nahvijou A, Hadji M, Rashidian H, Seyyedsalehi MS, Nemati S, et al. (2017) Iran Cancer Statistics in 2012 and projection of cancer incidence by 2035. Basic \& Clinical Cancer Research, 9(3): 3-22.

4. Akbari ME, Khayamzadeh M, Khoushnevis S, Nafisi N, Akbari A (2008) Five and ten years survival in breast cancer patients mastectomies vs. breast conserving surgeries personal experience. Iranian Journal of Cancer Prevention, 1(2): 53-6.

5. Baskar R, Lee KA, Yeo R, Yeoh KW (2012) Cancer and radiation therapy: current advances and future directions. International Journal of Medical Sciences, 9(3): 193.

6. Onitilo AA, Engel JM, Stankowski RV, Doi SA (2015) Survival comparisons for breast conserving surgery and mastectomy revisited: community experience and the role of radiation therapy. Clinical Medicine and Research, 13(2): 65-73.

7. Cuaron JJ, MacDonald SM, Cahlon O (2016) Novel applications of proton therapy in breast carcinoma. Chin Clin Oncol, 5(4): 52.

8. Silverstein MJ, Fastner G, Maluta S, Reitsamer R, Goer DA, Vicini $F$, et al. (2014) Intraoperative radiation therapy: a critical analysis of the ELIOT and TARGIT trials. Part 2-TARGIT. Ann Surg Oncol, 21(12): 3793-9.

9. Williams NR, Pigott KH, Brew-Graves C, Keshtgar MR (2014) Intraoperative radiotherapy for breast cancer. Gland Surg, 3(2): 109-19.

10. Miyamoto DT and Harris JR (2011) Molecular predictors of local tumor control in early-stage breast cancer. Semin Radiat Oncol, 21(1): 35-42.

11. Yoshida T, Takei H, Kurosumi M, Ninomiya J, Ishikawa $Y$, Hayashi $Y$, et al. (2016) True recurrences and new primary tumors have different clinical features in invasive breast cancer patients with ipsilateral breast tumor relapse after breast-conserving treatment. Breast J, 16(2): 127-33.
12. Barros AC, Hanna SA, Carvalho HA, Martella E, Andrade FE, Piato JR, et al. (2014) Intraoperative full-dose of partial breast irradiation with electrons delivered by standard linear accelerators for early breast cancer. Int J Breast Cancer, 2014: 568136.

13. Sedlmayer F, Reitsamer R, Fussl C, Ziegler I, Zehentmayr F, Deutschmann H, et al. (2014) Boost IORT in Breast Cancer: Body of Evidence. Int J Breast Cancer, 2014: 472516.

14. Brown LC, Mutter RW, Halyard MY (2015) Benefits, risks, and safety of external beam radiation therapy for breast cancer. Int J Womens Health, 7: 449-58.

15. Coombs NJ, Coombs JM, Vaidya UJ, Singer J, Bulsara M, Tobias JS, et al. (2016) Environmental and social benefits of the targeted intraoperative radiotherapy for breast cancer: data from UK TARGIT-A trial centres and two UK NHS hospitals offering TARGIT IORT. BMJ Open, 6(5): e010703.

16. Sedlmayer F, Reitsamer R, Wenz F, Sperk E, Fussl C, Kaiser $\mathrm{J}$, et al. (2017) Intraoperative radiotherapy (IORT) as boost in breast cancer. Radiation Oncology, 12(1): 23.

17. Harris EE and Small Jr W (2017) Intraoperative radiotherapy for breast cancer. Frontiers in Oncology, 7: 317.

18. Bravata V, Minafra L, Russo G, Forte GI, Cammarata FP, Ripamonti $M$, et al. (2015) High-dose ionizing radiation regulates gene expression changes in the MCF7 breast cancer cell line. Anticancer research, 35(5): 2577-91.

19. Shahani M, Shakeri J, Akbari ME, Arefnezhad B, Tafti A, Zali $\mathrm{H}$, et al. (2020) Transcriptomic and proteomic approaches reveal biological basis of intraoperative radiotherapytreated tumor bed modification in breast cancer patients: A pilot study. Journal of Proteomics, 212: 103596.

20. Baghani HR, Aghamiri SMR, Mahdavi SR, Akbari ME, Mirzaei HR (2015) Comparing the dosimetric characteristics of the electron beam from dedicated intraoperative and conventional radiotherapy accelerators. Journal of Applied Clinical Medical Physic, 16(2): 62-72.

21. Wen B, Zhou R, Feng Q, Wang Q, Wang J, Liu S (2014) IQuant: an automated pipeline for quantitative proteomics based upon isobaric tags. Proteomics, 14(20): $2280-$ 5.

22. Savitski MM, Wilhelm M, Hahne $H$, Kuster B, Bantscheff $M$ (2015) A scalable approach for protein false discovery rate estimation in large proteomic data sets. Molecular \& Cellular Proteomics, 14(9): 2394-404.

23. Minafra L, Bravata V, Russo G, Forte GI, Cammarata FP, Ripamonti M, et al. (2015)Gene expression profiling of MCF10A breast epithelial cells exposed to IOERT. Anticancer Research, 35(6): 3223-34.

24. Forte GI, Minafra L, Bravatà V, Cammarata FP, Lamia D, Pisciotta P, et al. (2017) Radiogenomics: the utility in patient selection. Translational Cancer Research, 6(S5): S852S74.

25. Paulsen GH, Strickert T, Marthinsen AB, Lundgren S (1996) Changes in radiation sensitivity and steroid receptor content induced by hormonal agents and ionizing radiation in breast cancer cells in-vitro. Acta Oncologica, 35(8): 10119. 
26. Bravatà V, Cava C, Minafra L, Cammarata F, Russo G, Gilardi $M$, et al. (2017) Radiation-induced gene expression changes in high and low grade breast cancer cell types. International Journal of Molecular Sciences, 19(4): 1084.
27. Porter JR, Fisher BE, Baranello L, Liu JC, Kambach DM, Nie $Z$, et al. (2017) Global inhibition with specific activation: how p53 and MYC redistribute the transcriptome in the DNA double-strand break response. Molecular Cell, 67(6): 1013-25. e9. 